\title{
Acute effects of ambient ozone on mortality in Europe and North America: results from the APHENA study
}

\author{
Roger D. Peng, \\ Department of Biostatistics, Johns Hopkins Bloomberg School of Public Health, $615 \mathrm{~N}$ Wolfe St, \\ Baltimore, MD 21205, USA \\ Evangelia Samoli, \\ Department of Hygiene, Epidemiology, and Medical Statistics, University of Athens Medical \\ School, Athens, Greece \\ Luu Pham, \\ Department of Biostatistics, Johns Hopkins Bloomberg School of Public Health, 615 N Wolfe St, \\ Baltimore, MD 21205, USA \\ Francesca Dominici, \\ Department of Biostatistics, Harvard School of Public Health, Boston, MA, USA \\ Giota Touloumi, \\ Department of Hygiene, Epidemiology, and Medical Statistics, University of Athens Medical \\ School, Athens, Greece \\ Tim Ramsay, \\ McLaughlin Center for Population Health Risk Assessment, University of Ottawa, Ottawa, ON, \\ Canada \\ Richard T. Burnett, \\ McLaughlin Center for Population Health Risk Assessment, University of Ottawa, Ottawa, ON, \\ Canada \\ Environmental Health and Consumer Products Branch, Health Canada, Ottawa, ON, Canada \\ Daniel Krewski, \\ McLaughlin Center for Population Health Risk Assessment, University of Ottawa, Ottawa, ON, \\ Canada
}

Alain Le Tertre,

Environmental Health Department, National Institute of Public Health Surveillance (InVS), SaintMaurice Cedex, France

Aaron Cohen,

Health Effects Institute, Boston, MA, USA

Richard W. Atkinson,

Division of Population Health Sciences and Education, MRC-HPA Centre for Environment and Health, St George's, University of London, London, UK

\section{H. Ross Anderson,}

\footnotetext{
(C) Springer Science+Business Media B.V. 2012

Correspondence to: Roger D. Peng, rpeng@ jhsph . edu.

The authors state that they have no conflicts of interest.
} 
Division of Population Health Sciences and Education, MRC-HPA Centre for Environment and Health, St George's, University of London, London, UK

Environmental Research Group, MRC-HPA Centre for Environment and Health, King's College, London, UK

Klea Katsouyanni, and

Department of Hygiene, Epidemiology, and Medical Statistics, University of Athens Medical

School, Athens, Greece

Jonathan M. Samet

Department of Preventive Medicine, Keck School of Medicine, and USC Institute for Global

Health, University of Southern California, Los Angeles, CA, USA

Roger D. Peng: rpeng@jhsph.edu

\section{Abstract}

The "Air Pollution and Health: A Combined European and North American Approach" (APHENA) project is a collaborative analysis of multi-city time-series data on the association between air pollution and adverse health outcomes. The main objective of APHENA was to examine the coherence of findings of time-series studies relating short-term fluctuations in air pollution levels to mortality and morbidity in 125 cities in Europe, the US, and Canada. Multi-city time-series analysis was conducted using a two-stage approach. We used Poisson regression models controlling for overdispersion with either penalized or natural splines to adjust for seasonality. Hierarchical models were used to obtain an overall estimate of excess mortality associated with ozone and to assess potential effect modification. Potential effect modifiers were city-level characteristics related to exposure to other ambient air pollutants, weather, socioeconomic status, and the vulnerability of the population. Regionally pooled risk estimates from Europe and the US were similar; those from Canada were substantially higher. The pooled estimated excess relative risk associated with a $10 \mu \mathrm{g} / \mathrm{m}^{3}$ increase in $1 \mathrm{~h}$ daily maximum $\mathrm{O}_{3}$ was $0.26 \%$ (95\% CI, $0.15 \%, 0.37 \%)$. Across regions, there was little consistent indication of effect modification by age or other effect modifiers considered in the analysis. The findings from APHENA on the effects of $\mathrm{O}_{3}$ on mortality in the general population were comparable with previously reported results and relatively robust to the method of data analysis. Overall, there was no indication of strong effect modification by age or ecologic variables considered in the analysis.

\section{Keywords}

Ozone; Mortality; Time-series; Multi-city; Cardiovascular; Respiratory

\section{Introduction}

The "Air Pollution and Health: A Combined European and North American Approach" (APHENA) project is a collaboration among the investigators carrying out the multi-city European APHEA (Air Pollution and Health: A European Approach) project, the US NMMAPS (National Morbidity, Mortality and Air Pollution Study), as well as multi-city Canadian studies (Katsouyanni et al. 2009). The APHENA project focuses on the short-term health effects of particulate matter less than $10 \mu \mathrm{g}$ in aerodynamic diameter $\left(\mathrm{PM}_{10}\right)$ and ozone $\left(\mathrm{O}_{3}\right)$ on daily mortality and hospital admissions. The project originated at a time when the results of the multi-city analyses, including those of APHEA and NMMAPS, were being reported and their findings used in the establishment of ambient air quality standards for particulate matter. Subsequently, the results of these multi-city studies have been cited in the development of air quality standards and guidelines for $\mathrm{O}_{3}$ (US Environmental Protection 
Agency 2006; World Health Organization 2006). The primary objective of the project was to develop a standardized methodology to assess the sensitivity of findings to analytical methods and to examine the coherence of findings on the short-term effects of air pollution on health in multicity studies carried out in Europe and North America. Further details of the APHENA project are described in a technical report available from the Health Effects Institute (Katsouyanni et al. 2009).

This paper provides the APHENA findings with regard to the association between daily concentrations of $\mathrm{O}_{3}$ and all-cause, cardiovascular, and respiratory mortality. A prior report provides the findings on $\mathrm{PM}_{10}$ (Samoli et al. 2008). Using the APHENA data, we were able to explore heterogeneity in the effect of $\mathrm{O}_{3}$ on mortality across the broad range of atmospheres included in the APHENA cities. Any consideration of heterogeneity needs to address the extent to which apparent heterogeneity across cities reflects the consequences of differing data collection and analytic methodologies. Based on past work by the APHENA investigators and on extensive sensitivity analysis, we developed uniform approaches for the first-stage (within-city) analyses of the time-series data used in earlier reports (Peng et al. 2006; Touloumi et al. 2006). The first-stage regression coefficients were then used in second-stage analyses aimed at obtaining pooled estimates and characterizing heterogeneity of the effect of $\mathrm{O}_{3}$ across the APHENA cities.

\section{Materials and methods}

\section{Data}

The APHENA project used already assembled databases for the first-stage analyses. For the $\mathrm{O}_{3}$ analysis, we used the daily $1 \mathrm{~h}$ maximum $\mathrm{O}_{3}$ concentration. The combined data included 86 US cities in NMMAPS (of which 50 provided year-round full time-series data and 36 provided summer-only data); 23 European cities in the APHEA project (that provided full time-series data); and 12 Canadian cities (that provided full time-series data) used in previous multi-city projects and selected on availability of air pollution monitoring data. The databases included daily counts of all-cause mortality (excluding deaths from external causes, International Classification of Disease, ninth revision (ICD-9)>800) for all ages and stratified by those above and under 75 years of age. Daily air pollution measurements were obtained from monitoring networks established in each city. City-specific time-series data on daily temperature $\left({ }^{\circ} \mathrm{C}\right.$, daily mean) were used to control for the potential confounding effects of weather.

\section{Methods}

The APHENA investigators implemented a new protocol for re-analysis of the daily air pollution data from the European and North American cities, using a hierarchical modeling approach. This protocol differed from the protocols originally used to analyze the US, European, and Canadian databases. First, regression models were fit in each city separately to control for seasonal effects, weather, and other potential confounders. Based on extensive simulation results, two methods were considered for smoothing: the natural spline (NS) approach for parametrically modelling flexible families of curves and the penalized regression spline (PS) approach, as implemented in the mgcv R package (Wood 2004).

In the initial methodological exploration, the number of degrees of freedom (df) for seasonality control was found to be the most important parameter in model specification with respect to the size of the effect estimate (Katsouyanni et al. 2009). Sensitivity analyses were carried out by varying the dfs used to control for seasonality (Peng et al. 2006; Touloumi et al. 2006). We used the estimated effect parameters from the individual city 
analysis in second-stage models to provide center-specific (Canada, Europe, and the US) and overall estimates and to investigate potential effect modifiers.

The year-round $\mathrm{O}_{3}$-mortality associations for each city were described using log-linear Poisson regression models allowing for overdispersion. The city-specific Poisson regression model is of the form:

$$
\log E\left[Y_{t}^{c}\right]=\alpha^{c}+\beta^{c} O_{3 t}^{c}+s^{c}(t, k)+\sum_{i} s_{i}^{c}\left(x_{i t}^{c}, k_{i}\right)+[\text { others }]
$$

where $E\left[Y_{t}^{c}\right]$ is the expected value of the Poisson distributed variable $Y_{t}^{c}$ indicating the daily mortality count on day $t$ in city $c$ with $\operatorname{Var}\left(Y_{t}^{c}\right)=\varphi \mathrm{E}\left[Y_{t}^{c}\right], \varphi$ is the overdispersion parameter, $x_{i t}{ }^{c}$ is the value of the $x_{i}$ meteorological covariate on day $t$ at city $c$, and $O_{3 t}{ }^{c}$ is the air pollution level on day $t$ at city $c$. The smooth functions $s^{\mathcal{C}}$ capture the non-linear relationship between the time-varying covariates and calendar time and daily mortality. We used both PS and NS as smooth functions in the analysis. In this setting, $k_{i}$ is the number of basis functions for the ith smooth function, where we used natural splines as basis functions for the penalized regression splines. We also included dummy variables for day of the week and bank holiday effects.

The smooth function of time serves as a proxy for any time-dependent outcome predictors or confounders with long-term trends and seasonal patterns not explicitly included in the model. These functions remove long-term trends and seasonal patterns from the data to guard against confounding by smoothly varying unmeasured temporal confounders. We fit separate models with 3, 8, and $12 \mathrm{dfs}$ per calendar year of data in the smooth function of time. Minimization of the sum of the absolute values of the partial autocorrelation function (PACF) of the model's residuals was also used as another criterion to select the optimal number of dfs, when the PS method was applied. The minimum number of dfs, allowed under PACF, was $3 \mathrm{dfs}$ per year. The dfs chosen by the PACF were then used to fit a NS model. Hence, with the minimum PACF procedure, the model for each city uses a different df depending on the data for that city. To control for weather, we included smooth terms of temperature on the day of death and the day before death in the models. The dfs for both temperature terms were set to 3. For models based on minimization of PACF criterion, autoregressive terms were introduced if significant autocorrelation remained in the residuals for the final model. We did not control for influenza epidemics because these data were not generally available for all locations and since previous results have shown that these do not bias the association between air pollution and mortality (Braga et al. 2000; Touloumi et al. 2005).

We included $\mathrm{O}_{3}$ in the models under different lag structures. In particular, we used the average of the same and previous day's air pollution as a predictor of increased mortality and also assessed the effect of the previous day's air pollution (lag 1). We also applied unconstrained distributed lag models spanning lags 0,1 , and 2 . When fitting distributed lag models, we used the same distributed lag terms for temperature as for $\mathrm{O}_{3}$. To investigate potential confounding effects by $\mathrm{PM}_{10}$, we applied two-pollutant models that controlled for the effects of concomitant exposure to particulate air pollution.

We also carried out center-specific threshold analyses to investigate the exposure-response relationship between $\mathrm{O}_{3}$ and all-cause mortality separately in Europe, the US, and Canada. For computational expediency, we used models with NS and $8 \mathrm{df}$ per year for control of seasonal confounding. We selected a grid of threshold values, ranging from 0 to $75 \mu \mathrm{g} / \mathrm{m}^{3}$ in increments of $5 \mu \mathrm{g} / \mathrm{m}^{3}$ (i.e., $0,5,10$, up to $75 \mu \mathrm{g} / \mathrm{m}^{3}$ ). For each threshold value $h$, we fit a 
threshold model to the data for the available cities. In the threshold model, we included a pollutant term $(x+)$ in the model of the form (pollutant- $h$ )+, where $x+=x$ if $x \geq$ and $x+=0$ if $x<0$, where $h$ is the threshold value. We then computed the Akaike information criterion (AIC) value of the fitted model for all cities within each center for a given threshold value and then the average AIC for that threshold over all cities in the center. We repeated the analysis for all threshold values and set a possible threshold at the value that minimized the mean AIC.

For the second stage of the analysis, we assumed the city-specific effects $\beta^{c}$ to be normally distributed around an overall effect. To test whether variability in the effects $\beta^{c}$ was explained by city-specific characteristics, we estimated pooled regression coefficients using a Bayesian hierarchical model of the city-specific effects $\beta^{c}$ on potential city-level effect modifiers, with weights inversely proportional to the variances of the estimates $\beta^{c}$. For this analysis, the coefficients $\beta^{c}$ were taken from the models using PS and $8 \mathrm{df}$ per year in the smooth function of time. These models assumed that city-specific effects $\beta^{c}$ were a sample of independent observations from a normal distribution with the same mean and with variances equal to the between-cities variance and the squared standard error of $\beta^{c}$. As a special case, we pooled the city-specific risk estimates with an intercept only to obtain region-specific average risk estimates.

A set of potential effect modifiers was collected to describe variation in the ozone effects across locations. Because of the substantial challenges in identifying measures with sufficient commonality across all three regions, the list of potential variables was necessarily limited. Effect modifiers used in the analysis included variables describing the average air pollution level and mix in each city $\left(\mathrm{NO}_{2}\right.$ coefficient of variation, mean $\mathrm{SO}_{2}, \mathrm{O}_{3}$ coefficient of variation, and the ratio of $\mathrm{NO}_{2}$ to $\mathrm{PM}_{10}$ ), the health status of the population, the geographical area, and the climatic conditions (Katsouyanni et al. 2009). The only indicator of socioeconomic status that was available and comparable between cities was the percent unemployment rate. Based on exploratory analysis, we examined potential effect modification patterns only for cities with complete time-series data and for the effects of the average of 2 days air pollution (lags 0 and 1). Since there were differences in the distribution of the effect modifiers between Europe and US, the analyses were location-specific.

\section{Warm season models}

In addition to evaluating the annual time-series data, the effects of $\mathrm{O}_{3}$ were also analyzed using the warm season (April-September) data only. In the models with only half-year data, dummy variables for the months (by year) instead of splines were used to control for temporal trends. Following extensive sensitivity analysis on the effect of temperature on summer $\mathrm{O}_{3}$ estimates, we decided to apply different degrees of smoothing for temperature depending on the smooth function included in the model. Hence, when we used NS as smoothing function, we allowed for $2 \mathrm{dfs}$ for same-day temperature and a linear term for previous-day temperature. When we used PS as the smoother, we used 3 dfs for same-day temperature and a linear term for lag 1 temperature.

\section{Results}

Overall, the main year-round analysis included 23 cities from Europe, 50 cities from the US, and 12 cities from Canada. For Canada, the cities ranged in population from 100,000 to over 2 million, with data from 1987 to 1996 . The daily total number of deaths from all causes (excluding accidents) ranged from 3 to 49 per day. The European cities contributed data for periods of 3 to 7 years between 1990 and 1997. Their populations ranged from slightly above 200,000 to about 7 million (The Netherlands was considered as one urban area because of its high density and urban character). The daily numbers of deaths from all 
causes ranged from 6 to 347. The complete US database included 86 cities with the largest populations in the US, spanning the period 1987 through 1996. There was substantial variation in population sizes (from about 250,000 to above 9 million) and related variation in the daily numbers of deaths, with means ranging from 5 to 198 . In the complete database, 50 cities had year-round monitoring of $\mathrm{O}_{3}$ and were used for the main analysis. For the analysis of data in the warm season only, all 86 cities were used. There was a wide variability in the median $\mathrm{O}_{3}$ levels in the European cities (ranging from 28.0 to $82.0 \mu \mathrm{g} / \mathrm{m}^{3}$ ) but less in the US (13.0 to $38.0 \mu \mathrm{g} / \mathrm{m}^{3}$ ) and Canadian cities (6.6 to $9.8 \mu \mathrm{g} / \mathrm{m}^{3}$ ).

The estimated effect of a $10 \mu \mathrm{g} / \mathrm{m}_{3}$ increase in the average of lags $0-1 \mathrm{O}_{3}$ on the total number of deaths for all ages is shown in Fig. 1 for a range of degrees of freedom for the smooth function of time. Across all three regions, the pooled estimated excess relative risk associated with a $10 \mu \mathrm{g} / \mathrm{m}^{3}$ increase in $1 \mathrm{~h}$ daily maximum $\mathrm{O}_{3}$ was $0.26 \%$ (95\% CI, $0.15 \%$, $0.37 \%$ ) for the model using natural splines and $8 \mathrm{df}$ per year. The effects for Canada are generally larger but with wider confidence intervals due to the smaller sizes of the Canadian cities. With the exception of estimates based on $3 \mathrm{df}$ per year, the estimates for Europe and the US are comparable. With natural splines with $8 \mathrm{df}$ per year, the estimates for Europe and the US show an increase of approximately $0.25 \%$ in mortality for a $10 \mu \mathrm{g} / \mathrm{m}^{3}$ increase in $\mathrm{O}_{3}$. The overall estimate combines each of the effects across the three centers. Because of the much higher uncertainty exhibited in the Canadian estimates, the overall effects largely reflect the contributions of the European and US estimates.

Table 1 presents the results of the year-round effect of $\mathrm{O}_{3}$ on total mortality all ages and by age group under the different lag structures examined. We present the results from models using $8 \mathrm{df}$ per year for seasonality control and PS, since we consider these to be relatively conservative estimates representing a reasonably justifiable control for unmeasured confounding. There was a statistically significant effect of $\mathrm{O}_{3}$ on total mortality for all ages in all three centers. In Europe and the US, the largest effects were observed when we took into account the exposure over 3 days for all the health outcomes analyzed, whereas the highest effects in Canada were observed when we took into account the average of lags $0-1$. In Canada and the US, the largest effect of annual $\mathrm{O}_{3}$ was observed in the older age group; in Europe, the largest effect was seen among those $<75$ years of age. There was some indication of confounding by $\mathrm{PM}_{10}$ in the Canadian results, but the estimates with and without $\mathrm{PM}_{10}$ in the models are not directly comparable for Canada, since $\mathrm{PM}_{10}$ measurements were only measured every sixth day. Correlations between $\mathrm{PM}_{10}$ and $\mathrm{O}_{3}$ were generally modest ranging from -0.40 to 0.55 across all three regions.

Table 2 presents the results from the analysis of the $\mathrm{O}_{3}$ and mortality data using only the warm period starting in April and going through September. Using these warm months only, the percent increase in all-cause non-accidental mortality for all ages associated with at 10 $\mu \mathrm{g} / \mathrm{m}^{3}$ increase in the average of lags $0-1 \mathrm{O}_{3}$ was $0.25 \%$ (95\% CI, $0.10 \%, 0.40 \%$ ) for Europe, $0.95 \%$ (95 \% CI, $0.65 \%, 1.2 \%$ ) for Canada, and $0.57 \%$ (95 \% CI, $0.35 \%, 0.79$ $\%$ ) for the US. All $\mathrm{O}_{3}$ effects were statistically significant during the warm period and higher than the annual effects. The same lag structure observed for the annual analysis is also present in the summer-only results.

The annual estimated effects of $\mathrm{O}_{3}$ on cardiovascular mortality for those above or below 75 years of age (data not shown) were generally slightly higher than those for total mortality. The US effect estimates were not statistically significant. The highest effect of $\mathrm{O}_{3}$ in Europe was observed on cardiovascular mortality among those $<75$ years using the average of 2 days exposure $(0.35 \%, 95 \% \mathrm{CI}, 0.12 \%, 0.58 \%)$. The highest effects on cardiovascular mortality in Canada were seen among the elderly using DL models $(1.20 \%, 95 \%$ CI, $0.18 \%$, 
$2.30 \%)$. The results for respiratory mortality were less consistent and not statistically significant in any case.

The summer-only $\mathrm{O}_{3}$ effect estimates for cause-specific mortality were positive (except for cardiovascular mortality among those $<75$ years of age in Canada) and in most cases statistically significant. In Canada, the highest effects of summertime $\mathrm{O}_{3}$ were observed for respiratory mortality for all ages under all lag structures considered while, in Europe and the US, the same was true only using lag $1 \mathrm{O}_{3}$ exposure.

Effect modification of the log-relative risks of $\mathrm{O}_{3}$ was assessed with respect to city-specific characteristics that had a uniform meaning across locations (Table 3). In general, there were no strong patterns of effect modification by these characteristics either within or across regions. In the US, temperature had a strong negative modifying effect, with the percent increase in mortality for a $10 \mu \mathrm{g} / \mathrm{m}^{3}$ increase in $\mathrm{O}_{3}$ estimated to range from $0.27 \%$ to $0.00 \%$ between the 25 th and 75 th percentiles of temperature. However, temperature did not modify the effect of $\mathrm{O}_{3}$ on mortality in either Europe or Canada. The coefficient of variation for $\mathrm{O}_{3}$ was associated with mortality in both the US and Europe, but not in Canada. Although there was some evidence that the mean $\mathrm{SO}_{2}$ level and the percent of the population over age 75 years modified the $\mathrm{O}_{3}$ effect, the evidence was not consistent across the US, Canada, and Europe.

Investigation of the exposure-response relationship between $\mathrm{O}_{3}$ and total mortality across all ages in APHENA - did not support the presence of a threshold in any of the three centers. If a threshold was present, we would expect to see a U-shaped curve when we plot the AIC values for the various threshold models against the thresholds used, with the minimum AIC value corresponding to the threshold. In fact, within each center, the cityspecific AIC plots were quite flat for most cities (data not shown).

\section{Discussion}

The APHENA project was motivated by the need for comparison of findings among three major time-series studies of air pollution and mortality and morbidity (APHEA, NMMAPS, and the Canadian multi-city project) and the possibility of exploring the basis for any heterogeneity among the risk estimates in these data sets. Its conduct required initial methodological work to establish a common analytic protocol for both first-stage and second-stage analyses that would replace the differing approaches of the original analyses (Katsouyanni et al. 2009). Additionally, a set of variables was developed across the three databases for consideration as potential effect modifiers in a second-stage analysis intended to explore determinants of heterogeneity. In APHENA, the first-stage results generally replicated the previous independent analyses by the three groups of investigators (Bell et al. 2004; Burnett et al. 1998, 2004; Gryparis et al. 2004). For $\mathrm{O}_{3}$ and mortality, there was some variation in findings by whether results were considered for the full year or only for the summer months, pointing to higher effects when only the warm period was considered. Mortality risk estimates for $\mathrm{O}_{3}$ tended to decrease with increasing $\mathrm{df}$ in the smooth function of time and were generally higher for the average of lags 0 and 1 compared with lag $1 \mathrm{O}_{3}$ concentration alone. The Canadian, APHEA, and NMMAPS data sets have now been extensively analyzed, and, while the quantitative risk estimates have varied across the three data sets, these data sets consistently demonstrate an acute effect of $\mathrm{O}_{3}$ on mortality.

In previously reported analyses, the European and North American risk estimates were quite similar, whereas the estimates based on the Canadian data were somewhat higher (Health Effects Institute 2003). Because of the common data analytic approach used in APHENA in all centers and cities, the higher values observed in Canada cannot be attributed to different 
analytic approaches. We note that $\mathrm{O}_{3}$ concentrations were lowest in the Canadian data and also covered the narrowest range. Consideration needs to be given to the possibility that the pollution mixture for which $\mathrm{O}_{3}$ is the indicator differs for Canada versus the United States and Europe. In particular, other co-varying pollutants that were not extensively examined here may explain some of the increased effect in Canada in a manner that is not demonstrated in the United States and Europe. There might also be greater residual confounding in the Canadian data from unmeasured seasonal or meteorological factors that were not adequately controlled for in the modeling. However, given the extensive work done and the specific focus of the APHENA group on developing models to adjust for potential confounding, this explanation carries less weight. Higher risk estimates could also come from having more accurate exposure and outcome data in Canada, as compared with the European countries and the US, but this possibility also seems unlikely, and we have no validation data available to pursue this conjecture.

For the pooled estimates of $\mathrm{O}_{3}$ risk in Europe and the US, there appeared to be some sensitivity to the amount of smoothing used in the smooth function of time, particularly when a small number of $\mathrm{df}$ was used. This sensitivity was most pronounced in US results, when penalized splines (PS) rather than natural splines (NS) were used. It should be noted that, even with the same number of degrees of freedom, the PS and NS methods are not identical because the PS method is nonparametric and the NS method is parametric. The discrepancy in the results for $3 \mathrm{df} /$ year between the NS and PS approaches has a theoretical basis related to the tradeoff between the parametric and nonparametric components of the PS models in explaining variation in the outcome variable (Peng et al. 2006; Speckman 1988). For the PS models with a small number of degrees of freedom, it is likely that there is insufficient adjustment for potential seasonal confounding in the relationship between ozone and mortality.

The APHENA findings confirm that $\mathrm{O}_{3}$ is associated with increased mortality in North America and Europe. The higher risks observed during the summer have been noted previously (Gryparis et al. 2004) and may reflect the higher concentrations during that season, greater personal exposure during the summer, and differing characteristics of the air pollution mixture by season. There was no consistent pattern of effect modification for the association between $\mathrm{O}_{3}$ and mortality. Nevertheless, we were able to identify stronger effect modification patterns in the US and some less pronounced patterns in the other two regions. The strong modifying effect of temperature on $\mathrm{O}_{3}$ effects in the US may reflect the extensive air conditioning use during warm periods that would result in less exposure to outdoor ozone. A similar pattern has been observed with PM (Bell et al. 2009). The modification by age, with people $\geq 75$ years of age demonstrating higher risks than the general population in US and Canada, suggests that elderly people may be more susceptible to the effects of $\mathrm{O}_{3}$. The higher effects of $\mathrm{O}_{3}$ in cities with higher levels of $\mathrm{SO}_{2}$ in these regions may reflect the synergistic effects of the air pollution mixture. The modifying effect of $\mathrm{O}_{3}$ variation, where cities with larger variation present smaller effects in Europe and US, may reflect adaptation of the population to current $\mathrm{O}_{3}$ levels. The lack of evidence of any threshold in risk between $\mathrm{O}_{3}$ and mortality is of interest here given the quantity of data combined across the three APHENA centers to conduct this analysis. However, identifying a threshold in time-series models is exceedingly difficult, and numerous simulation studies bear this out (Katsouyanni et al. 2009). The key limitation here is not the small sample size, but rather the inherently small association between $\mathrm{O}_{3}$ and mortality that is being estimated. If a threshold were to exist, it would likely require far more data than even the APHENA group could assemble. The results presented here are consistent with previous findings in the US (Bell et al. 2006).

The principal limitations in interpreting the APHENA findings lie with the data available to the investigators. The data came from multiple countries and had not been collected 
according to a uniform protocol across Europe and North America; however, all data had been edited and had undergone a quality assessment audit as part of the APHENA project. In the United States and Canada, nationwide reference methods for air pollution data collection are in place, and deaths are captured and coded uniformly. The European data came from 24 countries and methods for their collection varied among the countries, although they were assembled and edited with a common protocol. Well-documented approaches were used to develop air pollution concentration values that would be as complete and uniform as possible. Because of the nature of the APHENA project, it was not possible to assemble a fully harmonized database for the first-stage analysis, given the decision to begin with the existing databases and the potential magnitude of the task in reconstructing all of the time-series, particularly those for the European cities.

Consequently, differences in the time-series data, as well as the possibility of differing measurement error structures across the three sets of data, remain as potential contributors to heterogeneity.

Exploration of determinants of variation in the effect of air pollution across the APHENA cities was a main objective of the study. While a number of potential effect modifiers have been identified, the APHENA analyses were limited in exploring effect modification by the restricted number of variables that were measured with sufficient commonality across the full data set. Basic demographic data were available, but the only socioeconomic indicator that extended across the full data set was the unemployment rate. Information was not available on the health status of the populations, or on the frequency of specific diseases that have been postulated as effect modifiers, such as diabetes. Similarly, data were not available on housing characteristics or the mix of air pollution sources across the APHENA cities.

APHENA is the largest multi-city study time-series study published to-date, but other multicity studies have recently estimated the association of short-term exposure to ozone and daily mortality and report results that are consistent with APHENA. Coordinated multi-city studies in four major Asian cities and 14 UK conurbations reported $0.38 \%$ and $0.30 \%$ increases in all-natural cause mortality, respectively, associated with a $10 \mu \mathrm{g} / \mathrm{m}^{3}$ increase in an 8-h average ozone (Health Effects Institute 2010; Pattenden et al. 2010).

Unlike meta-analysis, which combines summary measures of the risk associated with exposure to $\mathrm{O}_{3}$ in different cities from published studies, the APHENA approach makes use of the primary raw data to combine information across cities and centers using a harmonized protocol. The data can be analyzed with multiple models, so as to assess sensitivity of findings to model specification, and effect modification can be assessed using risk estimates coming from a common analytic approach. This approach removes potential heterogeneity due to differences in analytic methodology. Given these strengths of the approach, we would suggest that the periodic conduct of pooling exercises like APHENA is highly desirable. The ongoing assembly of standardized time-series data sets and data on potential effect modifiers into a single database so that analyses could be carried out periodically would also be beneficial. Such analysis can lead to more robust estimates of the population health risks of ambient air pollution that can be used to support risk management decisions in the area of air quality management (Craig et al. 2008).

\section{Acknowledgments}

Funding was provided through a contract with the Health Effects Institute. The views expressed in this paper are those of the authors and do not necessarily reflect the views of the Health Effects Institute (HEI) or its sponsors. 


\section{References}

Bell ML, McDermott A, Zeger SL, Samet JM, Dominici F. Ozone and short-term mortality in 95 US urban communities, 1987-2000. J Am Med Assoc. 2004; 292(19):2372-2378.

Bell ML, Peng RD, Dominici F. The exposure-response curve for ozone and risk of mortality and the adequacy of current ozone regulations. Environ Health Perspect. 2006; 114(4):532-536. [PubMed: 16581541]

Bell ML, Ebisu K, Peng RD, Dominici F. Adverse health effects of particulate air pollution: modification by air conditioning. Epidemiology. 2009; 20(5):682-686. [PubMed: 19535984]

Braga AL, Zanobetti A, Schwartz J. Do respiratory epidemics confound the association between air pollution and daily deaths? Eur Respir J. 2000; 16(4):723-728. [PubMed: 11106219]

Burnett RT, Cakmak S, Brook JR. The effect of the urban ambient air pollution mix on daily mortality rates in 11 Canadian cities. Can J Public Health. 1998; 89(3):152-156. [PubMed: 9654797]

Burnett RT, Stieb D, Brook JR, Cakmak S, Dales R, Raizenne M, et al. Associations between shortterm changes in nitrogen dioxide and mortality in Canadian cities. Arch Environ Health. 2004; 59(5):228-236. [PubMed: 16201668]

Craig L, Brook JR, Chiotti Q, Croes B, Gower S, Hedley A, et al. Air pollution and public health: a guidance document for risk managers. J Toxicol Environ Health A. 2008; 71(9-10):588-698. [PubMed: 18569631]

Gryparis A, Forsberg B, Katsouyanni K, Analitis A, Touloumi G, Schwartz J, et al. Acute effects of ozone on mortality from the "air pollution and health: a European approach" project. Am J Respir Crit Care Med. 2004; 170(10):1080-1087. [PubMed: 15282198]

Health Effects Institute. Revised analyses of time-series studies of air pollution and health. Cambridge: Health Effects Institute; 2003.

Health Effects Institute. Public Health and Air Pollution in Asia (PAPA): coordinated studies of shortterm exposure to air pollution and daily mortality in four cities, HEI research report 154 . Boston: Health Effects Institute; 2010.

Katsouyanni K, Samet JM, Anderson HR, Atkinson R, Le Tertre A, Medina S, et al. Air pollution and health: a European and North American approach (APHENA). Res Rep Health Eff Inst. 2009; 142:5-90. [PubMed: 20073322]

Pattenden S, Armstrong B, Milojevic A, Heal MR, Chalabi Z, Doherty R, Barratt B, Kovats RS, Wilkinson P. Ozone, heat and mortality: acute effects in 15 British conurbations. Occup Environ Med. 2010; 67:699-707. [PubMed: 20798017]

Peng RD, Dominici F, Louis T. Model choice in multi-site time series studies of air pollution and mortality. J R Stat Soc Ser A. 2006; 169(2):179-203.

Samoli E, Peng R, Ramsay T, Pipikou M, Touloumi G, Dominici F, et al. Acute effects of ambient particulate matter on mortality in Europe and North America: results from the APHENA study. Environ Health Perspect. 2008; 116(11):1480-1486. [PubMed: 19057700]

Speckman P. Kernel smoothing in partial linear models. J R Stat Soc Ser B. 1988; 50:413-436.

Touloumi G, Samoli E, Quenel P, Paldy A, Anderson RH, Zmirou D, et al. Short-term effects of air pollution on total and cardiovascular mortality: the confounding effect of influenza epidemics. Epidemiology. 2005; 16(1):49-57. [PubMed: 15613945]

Touloumi G, Samoli E, Pipikou M, Le Tertre A, Atkinson R, Katsouyanni K. Seasonal confounding in air pollution and health time-series studies: effect on air pollution effect estimates. Stat Med. 2006; 25(24):4164-4178. [PubMed: 16991105]

U.S. Environmental Protection Agency. Air quality criteria for ozone and related photochemical oxidants (final). EPA/600/R-05/004aF-cF. Washington, DC: U.S. Environmental Protection Agency; 2006.

Wood SN. Stable and efficient multiple smoothing parameter estimation for generalized additive models. J Am Stat Assoc. 2004; 99(467):673-686.

World Health Organization. Copenhagen: World Health Organization; 2006. Air quality guidelines: global ppdate 2005-particulate matter, ozone, nitrogen dioxide and sulfur dioxide. 


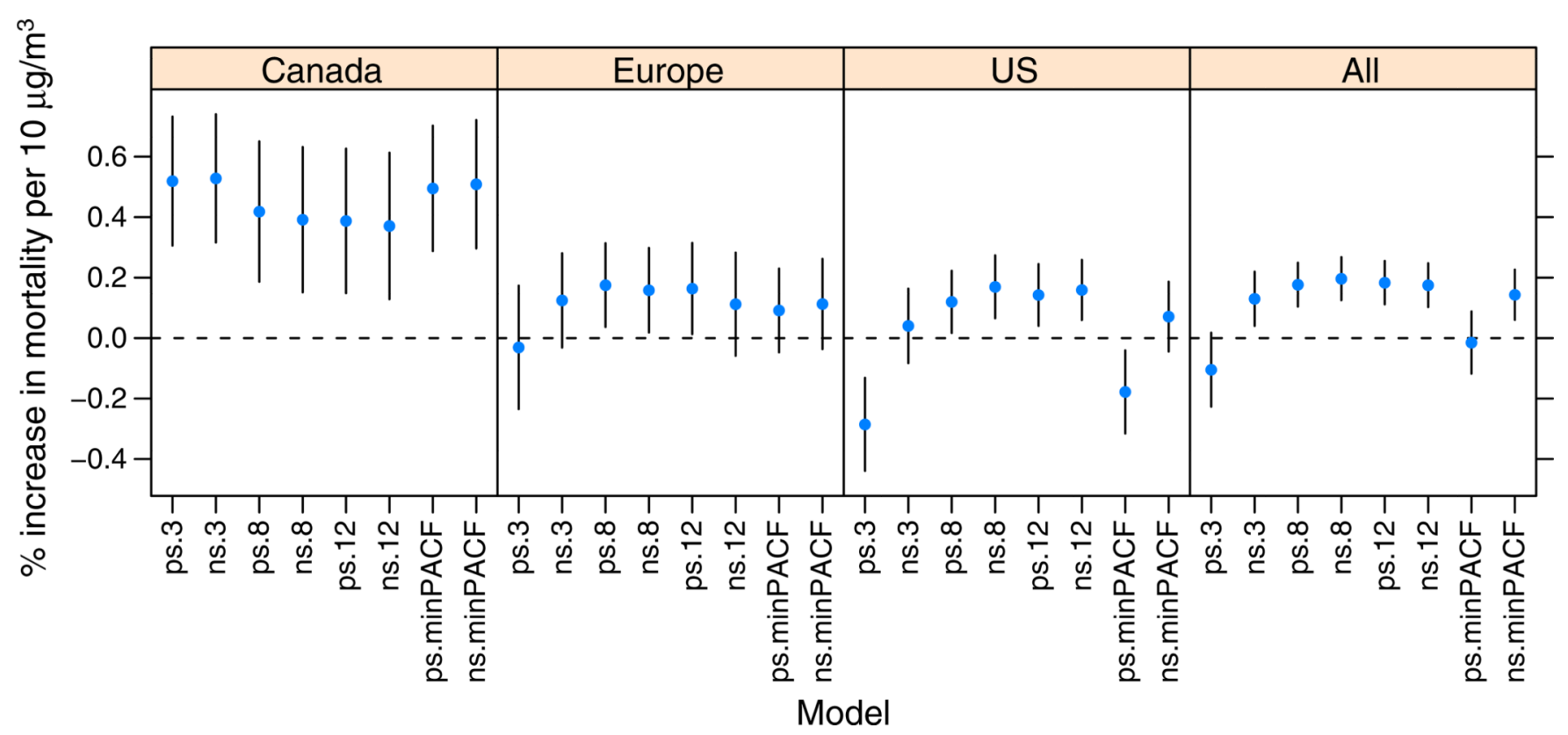

Fig. 1.

Effects of a $10 \mu \mathrm{g} / \mathrm{m}^{3}$ increase in $\mathrm{O}_{3}$ at the average of lags 0-1 on all cause mortality for three centers using NS and PS with 3, 8, 12, and minimum PACF degrees of freedom per year in the smooth function of time. $N S=$ natural spline; $P S=$ penalized regression spline; $P A C F=$ partial autocorrelation function 


\section{Table 1}

Percent increase $(95 \% \mathrm{CI}$ ) in the daily number of deaths (all ages, and $\geq 75$ and $<75$ years of age) associated with an increase of $10 \mu \mathrm{g} / \mathrm{m}^{3}$ in $\mathrm{O}_{3}$ concentrations (estimated by using $8 \mathrm{df} /$ year to control for seasonal patterns and PS) in the three centers for the whole year

\begin{tabular}{|c|c|c|c|c|}
\hline & \multicolumn{4}{|l|}{ Total mortality } \\
\hline & Average of lags 0-1 & Lag 1 & Lag 1 controlling for $\mathbf{P M}_{10}$ & Distributed lag (lags $0,1,2)$ \\
\hline \multicolumn{5}{|l|}{ All ages } \\
\hline Canada & $0.85(0.51,1.20)$ & $0.56(0.28,0.83)$ & $0.48(-0.18,1.20)$ & $0.75(0.25,1.30)$ \\
\hline Europe & $0.18(0.07,0.30)$ & $0.17(0.09,0.25)$ & $0.19(0.10,0.28)$ & $0.25(0.10,0.40)$ \\
\hline US & $0.31(0.09,0.52)$ & $0.18(0.00,0.35)$ & $0.13(-0.18,0.44)$ & $0.43(0.11,0.75)$ \\
\hline \multicolumn{5}{|c|}{$\geq 75$ years } \\
\hline Canada & $0.98(0.50,1.50)$ & $0.61(0.22,1.00)$ & $-0.07(-0.99,0.87)$ & $0.90(0.19,1.60)$ \\
\hline Europe & $0.12(-0.02,0.26)$ & $0.14(0.04,0.24)$ & $0.16(0.05,0.28)$ & $0.17(-0.03,0.37)$ \\
\hline US & $0.33(0.02,0.64)$ & $0.21(-0.04,0.46)$ & $0.12(-0.39,0.63)$ & $0.40(-0.05,0.85)$ \\
\hline \multicolumn{5}{|c|}{$<75$ years } \\
\hline Canada & $0.74(0.28,1.20)$ & $0.52(0.14,0.89)$ & $1.00(0.11,2.00)$ & $0.62(-0.06,1.30)$ \\
\hline Europe & $0.25(0.10,0.40)$ & $0.18(0.07,0.29)$ & $0.24(0.12,0.37)$ & $0.37(0.14,0.59)$ \\
\hline US & $0.30(0.04,0.56)$ & $0.15(-0.07,0.37)$ & $0.12(-0.33,0.56)$ & $0.50(0.09,0.92)$ \\
\hline
\end{tabular}

$O_{3}$ ozone, $P M_{10}$ particulate matter $<10 \mu \mathrm{m}$ aerodynamic diameter, $P S$ penalized splines 


\section{Table 2}

Percent increase $(95 \% \mathrm{CI}$ ) in the daily number of deaths (all ages, and $\geq 75$ and $<75$ years of age) associated with an increase of $10 \mu \mathrm{g} / \mathrm{m}^{3}$ in $\mathrm{O}_{3}$ concentrations (using PS for temperature) in the summer period

\begin{tabular}{|c|c|c|c|}
\hline & \multicolumn{3}{|l|}{ Total mortality } \\
\hline & Average of lags 0-1 & Lag 1 & Distributed lag (lags 0, 1, 2) \\
\hline \multicolumn{4}{|l|}{ All ages } \\
\hline Canada & $0.95(0.65,1.20)$ & $0.71(0.46,0.95)$ & $0.42(0.16,0.67)$ \\
\hline Europe & $0.25(0.10,0.40)$ & $0.29(0.19,0.39)$ & $0.34(0.16,0.53)$ \\
\hline US & $0.57(0.35,0.79)$ & $0.49(0.29,0.69)$ & $0.65(0.33,0.97)$ \\
\hline \multicolumn{4}{|c|}{$\geq 75$ years } \\
\hline Canada & $1.20(0.51,2.00)$ & $0.88(0.35,1.40)$ & $0.82(0.25,1.40)$ \\
\hline Europe & $0.23(0.07,0.39)$ & $0.30(0.17,0.44)$ & $0.32(0.08,0.57)$ \\
\hline US & $0.63(0.33,0.93)$ & $0.58(0.32,0.84)$ & $0.62(0.16,1.08)$ \\
\hline \multicolumn{4}{|c|}{$<75$ years } \\
\hline Canada & $0.60(0.20,1.00)$ & $0.45(0.12,0.78)$ & $0.42(0.16,0.67)$ \\
\hline Europe & $0.29(0.12,0.46)$ & $0.27(0.13,0.41)$ & $0.36(0.09,0.63)$ \\
\hline US & $0.50(0.22,0.78)$ & $0.40(0.16,0.64)$ & $0.70(0.28,1.12)$ \\
\hline
\end{tabular}

O3 ozone, $P S$ penalized splines 


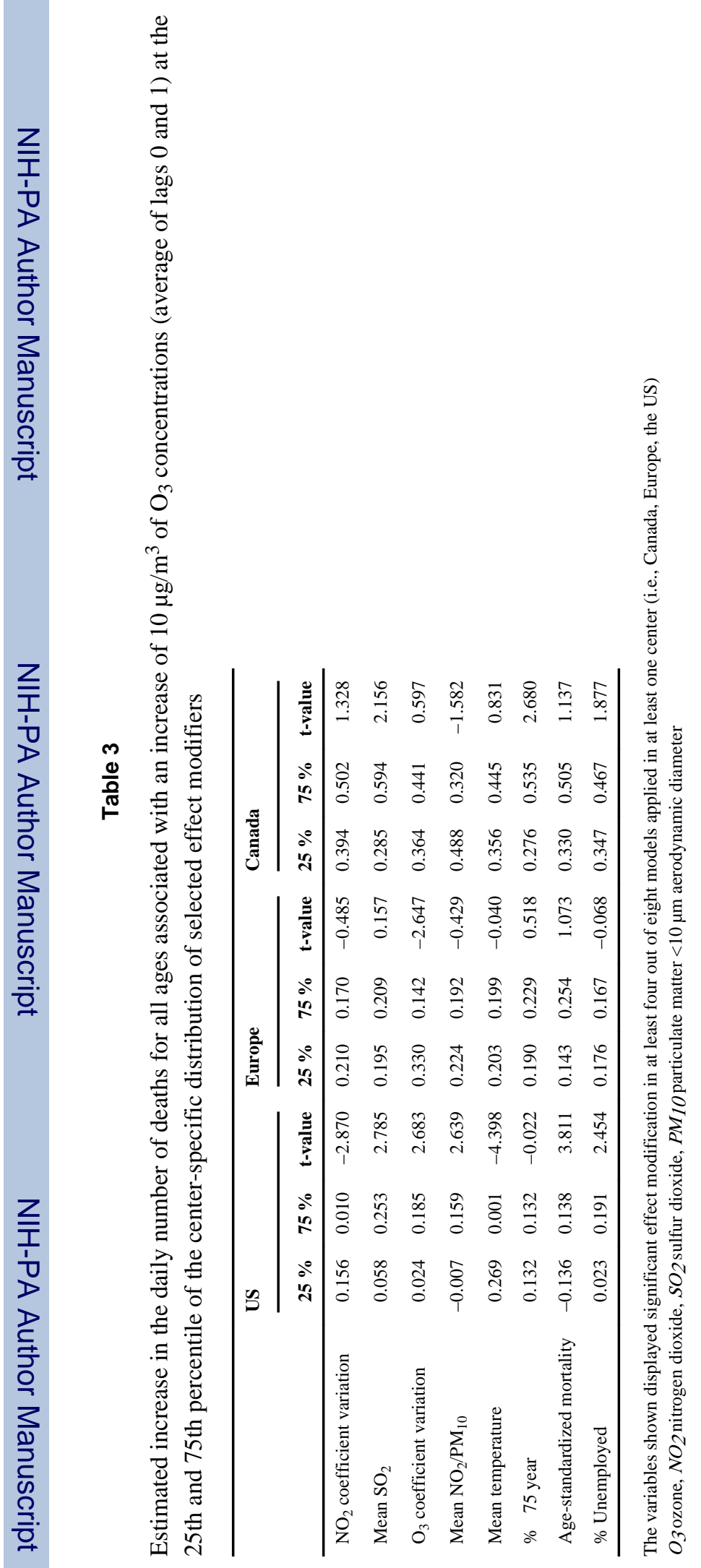

